\title{
Adolf Friedrich Fercher: a pioneer of biomedical optics
}

\author{
Christoph K. Hitzenberger
}




\title{
Adolf Friedrich Fercher: a pioneer of biomedical optics
}

\author{
Christoph K. Hitzenberger* \\ Medical University of Vienna, Center for Medical Physics and Biomedical Engineering, Vienna, Austria
}

\begin{abstract}
Adolf Friedrich Fercher, an outstanding pioneer of biomedical optics, passed away earlier this year. He was a brilliant and visionary researcher who pioneered various fields of biomedical optics, such as laser speckle flowgraphy, tissue interferometry, and optical coherence tomography (OCT). On the occasion of the 25th anniversary of OCT, this paper reviews and commemorates Fercher's pioneering work. $\odot$ The Authors. Published by SPIE under a Creative Commons Attribution 3.0 Unported License. Distribution or reproduction of this work in whole or in part requires full attribution of the original publication, including its DOI. [DOI: 10.1117/1.JBO.22.12.121704]
\end{abstract}

Keywords: biomedical optics; optical coherence tomography.

Paper 170561SSVR received Aug. 23, 2017; accepted for publication Oct. 26, 2017; published online Nov. 16, 2017.

\section{Introduction}

On March 10, 2017, Adolf Friedrich Fercher, an outstanding pioneer of biomedical optics, passed away. Fercher pioneered several fields of biomedical optics, such as laser speckle flowgraphy $(\mathrm{LSF})^{1}$ and ocular fundus pulsation measurements, ${ }^{2}$ but his most important work was certainly the introduction of partial coherence interferometry (PCI) as a tool for optical biometry of tissue and as a cornerstone of optical coherence tomography (OCT) ${ }^{3,4}$ Fercher et al. ${ }^{5}$ also pioneered the paradigm change in OCT technology from time-domain- to Fourier-domain OCT, improving the sensitivity and speed of OCT by 2 to 3 orders of magnitude, which laid the foundation for modern high-speed 3-D OCT imaging.

Another important legacy of Fercher was the foundation of the Vienna Biomedical Optics Group. Fercher was full of visionary ideas that were often many years ahead of his time. To bring these ideas to life required the formation of a strong group of researchers. A key to the success of this group was Fercher's way of selecting people with different scientific backgrounds, being a mentor for them, and giving them demanding tasks but letting them largely have a free hand in how to achieve their goals, so these scientists could identify themselves with their work and grow their own scientific reputation. This led to the formation of a very strong biomedical optics group in Vienna, which later subdivided into several individual groups.

It is the purpose of this review, on the occasion of the 25th anniversary of OCT, to commemorate Fercher's pioneering contributions to biomedical optics and especially to OCT. Because of spatial restrictions, this report is limited to the early pioneering work carried out at the University of Essen and to the initial phase of the Vienna group. More information on additional aspects of the work of the Vienna group can be found in a recent review. ${ }^{6}$

\section{Early Work}

Fercher's scientific career took an unusual path: after graduating in physics at Vienna University of Technology, he worked from

${ }^{*}$ Address all correspondence to: Christoph K. Hitzenberger, E-mail: christoph .hitzenberger@meduniwien.ac.at
1968 to 1975 at Carl Zeiss, Oberkochen, Germany, on optical testing, computer holography, and holographic interferometry. In 1975, he went from industry back to academia: from 1975 to 1986 as a professor at University of Essen, Germany, and since 1986 as a professor of medical physics at the Medical School of the University of Vienna, where he chaired the Department of Medical Physics until his retirement in 2008 (the Medical School of the University of Vienna was transformed into the independent Medical University of Vienna in 2004).

Fercher became interested in biomedical applications of optics at quite an early stage of his career. He published his first paper in this field when he was still working for Carl Zeiss. $^{7}$ In this paper, he applied Mie theory to calculate light scattering by a simplified cell model, consisting of spheres of different materials that mimic the cell nucleus and cell body. He showed that, for a dedicated receiver geometry, the scattered signal exhibits oscillations as a function of scattering angle, whereby the oscillation length is related to the particle diameter. Although he did not continue this work, it clearly shows his interest in biomedical optics at an early stage.

\subsection{Laser Speckle Flowgraphy}

After moving back from industry to academia, biomedical optics became the main focus of Fercher's work. The first field of research was related to his earlier work on speckle metrology: LSF. In cooperation, Fercher and Briers developed a method for analyzing retinal blood flow. ${ }^{1}$ The method is based on the following idea (cf. Fig. 1): a laser beam illuminates the retina via the lower half of the eye's pupil. The light is backscattered by the retina and imaged onto a photographic film via the upper part of the pupil. The random backscattering of the coherent light generates a speckle pattern on the image plane with well-defined speckle contrast. In areas of moving scatterers (e.g., red blood cells moving in retinal vessels), the speckle pattern will be blurred, leading to a reduced speckle contrast of the corresponding areas in the image plane. This effect can be exploited to generate angiographic images of the retina, identify retinal vessels, and, to a certain extent, quantify the velocity of blood flow. 


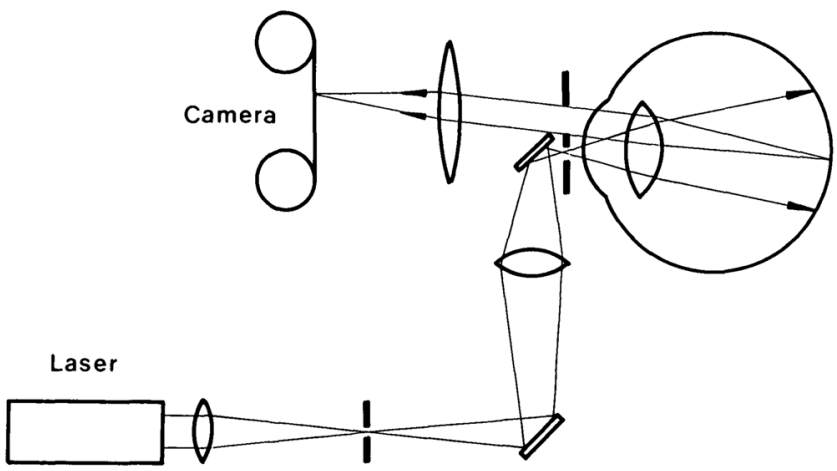

Fig. 1 Experimental arrangement for LSF of the retina. Reproduced with permission from Ref. 8, ( Association for Research in Vision and Ophthalmology.

Figure 2 shows retinal images acquired with this method. Figure 2(a) is the direct image, which does not show much detail. However, high-pass filtering of the image enhances the contrast, rendering the blurred areas of low speckle contrast (the vessels) dark [cf. Fig. 2(b)]. It should be mentioned here that digital image processing, as we would use it nowadays, was not easily available at that time. Instead, the high-pass filtering was based on an analog optical technique [using spatial filtering in a dark-field configuration to image the developed photographic film to generate the final image of Fig. 2(b)]. In this way, it was possible to generate first LSF angiograms of the human retina in the macula and optic nerve head area. ${ }^{8}$ However, the use of photographic film and the analog high-pass filtering made the whole procedure rather tedious and the optical high-
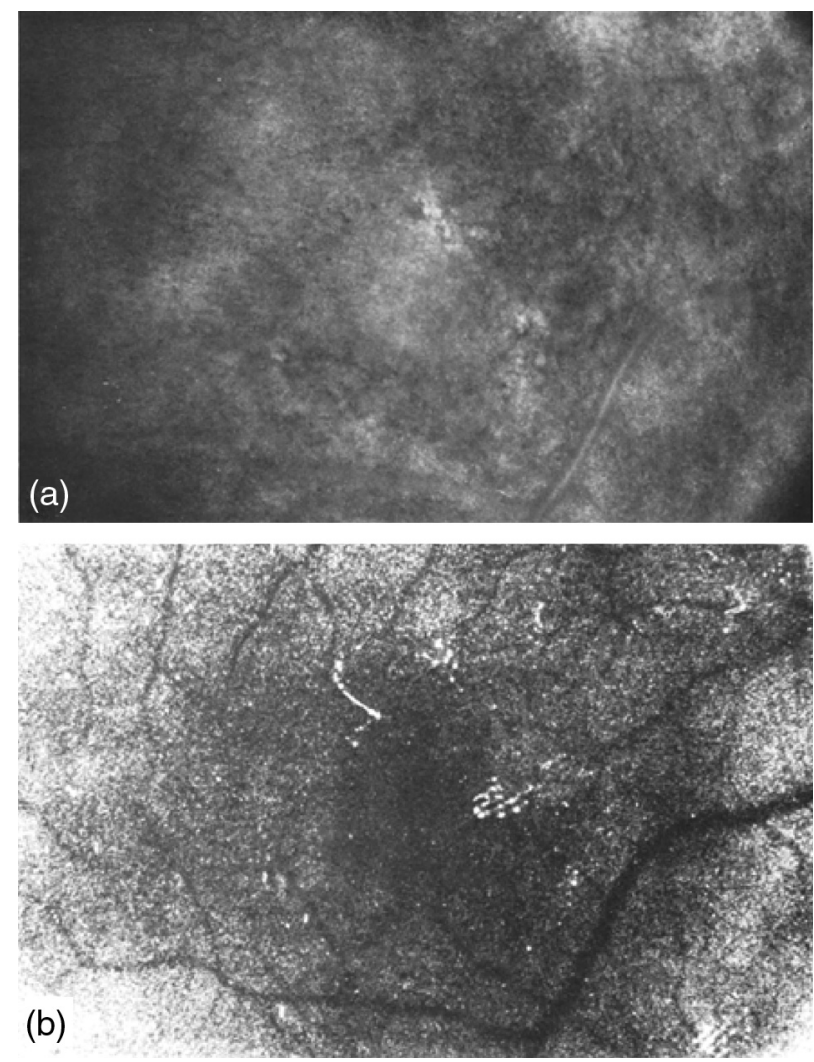

Fig. 2 LSF of the retina. (a) Direct image and (b) image after analog high-pass filtering. Reproduced with permission from Ref. 1, () Elsevier. pass filtering of structures on photographic film also generated additional artifacts (additional speckle caused by scattering within the film). This clearly indicates that the idea was many years ahead of its time.

A few years later, Fercher made a first step to improve the method: the photographic film was digitized and digital image processing was used to replace the analog high-pass filtering. This enabled the generation of color-coded flow maps. ${ }^{9}$ With the advent of modern, high-resolution and high-speed CCD and CMOS cameras of sufficient sensitivity in the 1990s and in the new millennium, the detour via photographic film became obsolete and LSF was revitalized. Meanwhile, it has found important applications, e.g., in cerebral blood flow imaging ${ }^{10,11}$ and in retinal blood flow imaging, ${ }^{12}$ the latter technology also being commercialized. ${ }^{13}$

\subsection{Interferometric Fundus Pulsation Measurements}

In the year after introducing LSF, Fercher et al. ${ }^{2}$ published another groundbreaking paper, demonstrating interferometric measurements of ocular fundus pulsations in the human eye. The paper is truly remarkable because it is based on an observation that later led to partial coherence interferometric ocular biometry, one of the cornerstones of OCT. The basic observation is shown in Fig. 3: the eye is illuminated by a HeNe laser via a beam splitter [Fig. 3(a)], and the light remitted by the eye is observed in transmission through the beam splitter. Within the light field emerging from the eye, concentric interference fringes [Fig. 3(b)] that pulsate synchronously with the subject's heartbeat can be observed. These fringes are generated by the light reflected at the cornea (which has a spherical wave front) and light backscattered by the retina (which is transformed into a plane wave by the refractive elements of the eye). By counting the interference fringes that pass through a single point of the image during a heartbeat cycle, the pulsation of the retina with respect to the cornea can be quantitatively measured. These pulsations provide information on the ocular blood flow.

Since a straightforward electronic counting of the interference fringes was prevented by noise, another method was used by Fercher's team: a slit stop that passed through the center of the fringe pattern was placed into the image plane; this slit was imaged onto a moving photographic film [cf. Fig. 4(a)]. This enabled recording of what we would now call an $M$-scan, the development of the interference fringes with time. Figure 4(b) shows an example of such a temporally changing interferogram. ${ }^{14}$ The vertical axis shows the spatial dimension along the slit; the horizontal axis indicates the time. By counting the interference fringes along the central horizontal line, the fundus pulsations can be measured. The diastolic phase of the heartbeat is characterized by widely separated (slowly moving) fringes, while the systolic phase is recognizable by narrow (rapidly moving) fringes (indicated by the filled triangle). The fundus pulsations measured by this method in healthy volunteers were on the order of a few micrometer, with a standard error (obtained over 10 heartbeat periods) of $\sim 0.1 \mu \mathrm{m}$. In an extension of this study, it could be shown that the fundus pulsations vary considerably with position of the reflection site on the retina, with the strongest pulsation amplitude of up to $\sim 20 \mu \mathrm{m}$ in the optic nerve head area. ${ }^{15}$

However, the necessity to use photographic film was again tedious and did not allow larger studies with this technique. Again, Fercher was way ahead of his time with this method. 

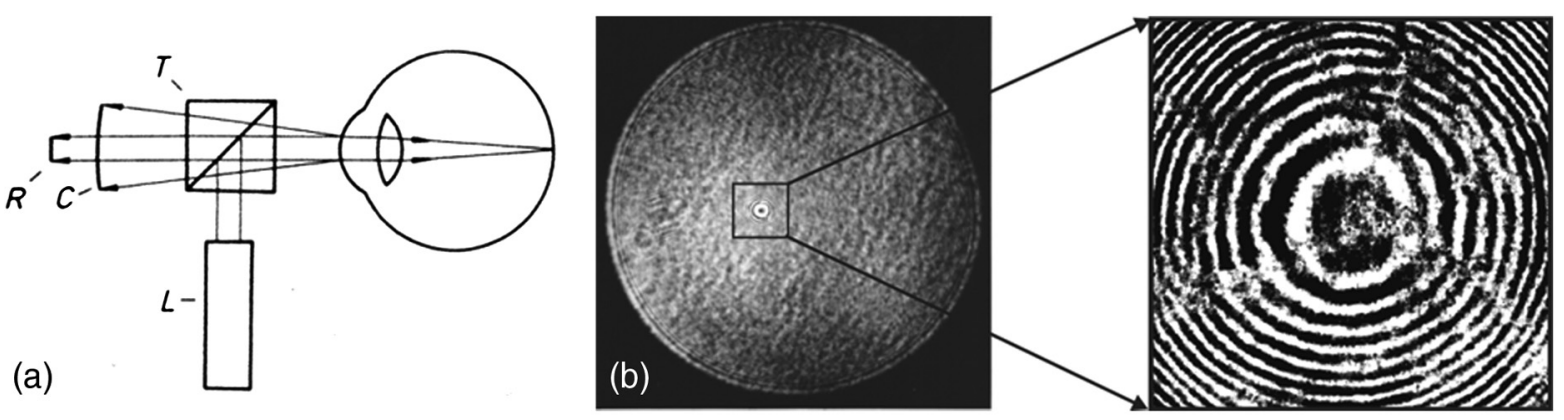

Fig. 3 Basic interference phenomenon for fundus pulsation measurements and partial coherence biometry. (a) Experimental arrangement and (b) interference pattern of beams reflected at the cornea and retina and zoom-in. $L$, laser; $T$, beam splitter; $C$, light reflected at the cornea; and $R$, light reflected at the retina. Reproduced with permission from Ref. 2 (a), () Taylor \& Francis and from Ref. 6 (b).
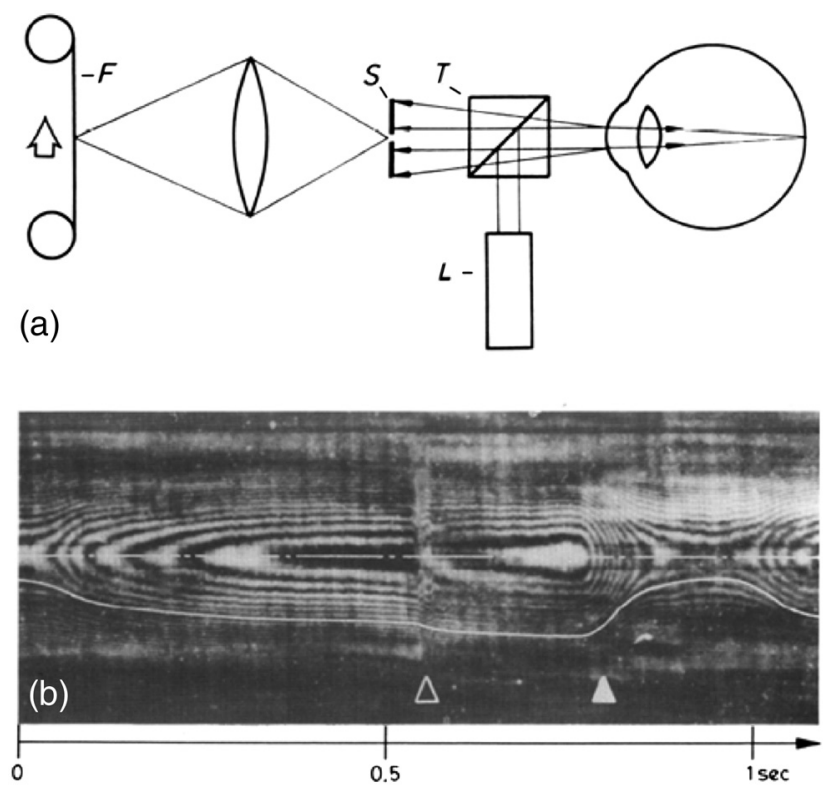

Fig. 4 Ocular fundus pulsation measurements. (a) Experimental arrangement and (b) development of interferometric fringes with time (along horizontal axis). $L$, laser; $T$, beam splitter; $S$, slit aperture; and $F$, film. Reproduced with permission from Ref. 2 (a), @ Taylor \& Francis; and from Ref. 14 (b), () SPIE.

More than a decade after the first demonstration of the method, this work was continued by Schmetterer, who had joined Fercher's group in Vienna. Schmetterer et al. ${ }^{16}$ replaced the moving photographic film by a CCD line scan camera, which had become available in the meantime, and coupled the interferometer to a fundus camera that allowed a precise control of the measurement location on the retina. This enabled the use of the method in numerous physiological and pharmaceutical studies investigating the influence of various stimuli and drugs on ocular blood flow in healthy subjects and patients with various diseases. ${ }^{17-19}$

\section{Coherence Ranging and Imaging}

\subsection{Partial Coherence Interferometry}

The observation of the interference pattern generated by light beams reflected at the cornea and retina also stimulated the idea that this phenomenon might be used for intraocular

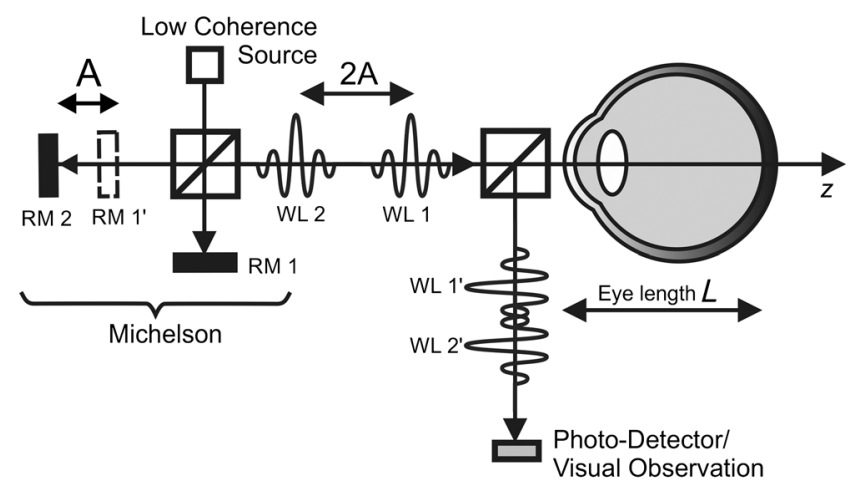

Fig. 5 Sketch of $\mathrm{PCl}$ intraocular ranging. $A$, interferometer path difference; RM, mirror; and WL, wavelet. Reproduced from Ref. 6.

ranging/ocular biometry. This idea can already be found in Fercher's first paper on fundus pulsation measurements. In the conclusion of his paper, Fercher states:. "In these measurements light with a rather long coherence length (of the order of $50 \mathrm{~mm}$ ) has to be used. When using light of limited coherence length, the path differences of the two reflected light beams must be compensated, for example by using a Michelson interferometer arrangement. Using this technique it might be possible to measure the optical length of the eye." In the same year, Fercher also filed a patent on that idea. ${ }^{20}$

The principle of the method is shown in Fig. 5. An external interferometer splits the beam of short coherence length into two components (WL1 and WL2) with a delay equal to twice the interferometer path difference $A$. These components illuminate the eye along a coaxial path and are each reflected at the cornea and retina. If $A$ equals the optical length of the eye $(L)$, wavelets WL1' and WL2', reflected at the retina and cornea, respectively, travel the same total path length and will overlap at the exit path, giving rise to a circular interference pattern similar to that of Fig. 3(b). To measure the length of the eye, the interferometer arm length is adjusted until the fringes become visible. The eye length can then be obtained from the easily measurable interferometer arm length difference $A$ by division by the (group) refractive index of the ocular media.

Again, this idea was ahead of its time: suitable light sources were not easily available in 1982 . The ideal light source should have a high spatial coherence (i.e., transversal monomode and Gaussian beam profile) and a very low temporal coherence (broad emission spectrum, preferably with a Gaussian spectral 
shape). The dye lasers that Fercher's group tried initially suffered from beam instabilities. In the mid-1980s, multimode laser diodes with a coherence length of $\sim 100 \mu \mathrm{m}$ became available, enabling a first report on axial eye length measurements by PCI in $1986 .{ }^{3}$ In a study on eight healthy volunteers, a repeatability (standard deviation) of $\sim 30 \mu \mathrm{m}$ was demonstrated, together with a good agreement with measurements by ultrasound. ${ }^{4}$

The targeted application of PCI axial eye length measurements was cataract surgery, where the opaque natural lens is replaced by an artificial intraocular plastic lens (IOL). The refractive power to be selected for that lens depends critically on the length of the eye, so a precise knowledge of this dimension is needed for the patient to be emmetropic after surgery. While the precision achieved by Fercher's initial approach of PCI biometry was very appealing, the measurement method was too slow for application in patients: it took about $15 \mathrm{~min}$ to measure the eye length by manual adjustment of the interferometer plates and visual search for interference fringes by the observer.

After moving to Vienna in 1986, Fercher established a new biomedical optics group to continue the work started in Essen. As the first member of the new group, the author of this review was charged with improving PCI ocular biometry to enable measurements in patients. The solution to the problem was to employ principles of heterodyne interferometry: one interferometer mirror is moved with a constant speed by a stepper motor, which causes a Doppler shift of the frequency of the corresponding beam component. In case of path length matching, an interferometric signal burst at the Doppler frequency is measured by a photodetector and extracted by bandpass filtering. ${ }^{21}$ The envelope of this signal is recorded as a function of path delay, and a signal peak is observed at every position where path length matching occurs. This signal became later known as an optical $A$-scan, a one-dimensional image that forms the basic element of an OCT dataset.

Figure 6(a) shows one of the first $A$-scans achieved with this method in a healthy human eye. ${ }^{21}$ Two signal peaks are observed here, indicating the positions of the anterior and posterior surface of the retina (with respect to the cornea as a reference surface). This $A$-scan provides not just the eye length but also the thickness of the retina. By adjusting the angle between vision axis and measurement direction, transverse information became available. Figure 6(b) shows a profile of the retinal surface acquired in that way. At an angle of $\sim-12 \mathrm{deg}$ to $-14 \mathrm{deg}$ (nasal), the excavation of the optic disc is observed.

In a next step, the multimode laser diode was replaced by a superluminescent diode, which improved the axial resolution by an order of magnitude. ${ }^{22}$ In a further improvement, Drexler et al. ${ }^{23}$ implemented a transverse scanning unit to automatically scan the retina. The combination of these improvements enabled numerous studies of high-precision measurements of various intraocular distances and of their change during accommodation. ${ }^{24,25}$

The most important application of PCI ocular biometry, however, was the one initially devised by Fercher: measurement of the axial length of cataract eyes. In a first study, 196 cataract eyes of 100 patients were measured, with a success rate of $>90 \% .^{26}$ This study demonstrated an excellent correlation with ultrasound measurements, with a superior precision of the optical method. Later studies demonstrated that the precision of PCI can be more than an order of magnitude better than that
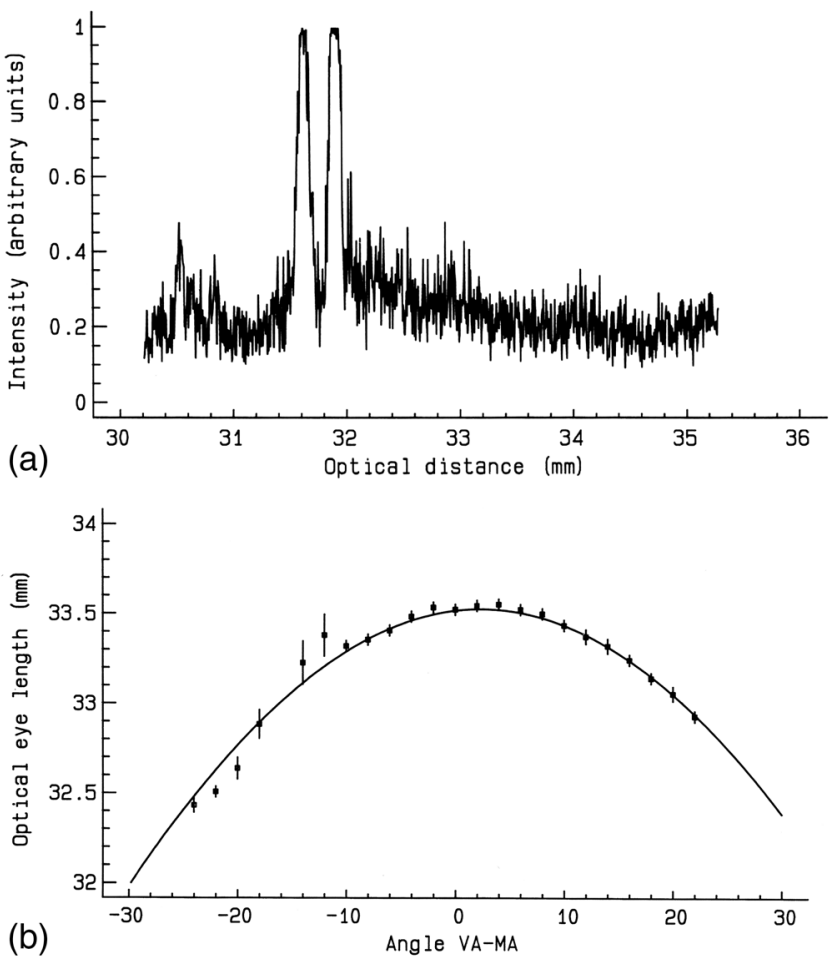

Fig. 6 (a) $\mathrm{PCl} A$-scan indicating axial eye length and retinal thickness and (b) fundus profile obtained by $\mathrm{PCl}$. VA, vision axis and MA, measurement axis. Reproduced with permission from Ref. 21, (c) Association for Research in Vision and Ophthalmology.

of ultrasound, ${ }^{27}$ which can provide an improved postoperative visual outcome of patients. ${ }^{28}$ More details on these and other ocular biometry studies can be found in a recent review. ${ }^{6}$

The improved precision and the contact-free application of PCI, which avoided anesthesia and any risk of infection, thereby being more convenient for the patient, finally convinced Carl Zeiss to market this technology as the "IOL Master." ${ }^{29}$ In the following years, this technology largely replaced ultrasound instruments for ocular biometry, and, with 10,000 s of instruments installed worldwide, it is now the gold standard for this application and one of the most successful biomedical optics technologies so far.

\subsection{Optical Coherence Tomography}

Huang et al. ${ }^{30}$ of Fujimoto's group at Massachusetts Institute of Technology were the first to synthesize optical $A$-scans to twodimensional images ( $B$-scans), thereby demonstrating the potential of this technology to record cross-sectional images of translucent and scattering tissue. In their seminal paper, they also coined the name "optical coherence tomography" for this new imaging modality. The first images from 1991 were recorded in vitro. Fercher was immediately convinced of this technique and strived to acquire in vivo images. The first in vivo OCT image recorded in a human retina by Fercher's group was published in $1993^{31}$ (a few weeks after the first in vivo image was published by the MIT group ${ }^{32}$ ). Figure 7 shows that image. Although the image quality is poor compared with modern standards, tissue features, such as retinal thickness, the excavation of the optic disc, and the lamina cribrosa, are visible. Since the sensitivity and measurement speed achieved by the technique used to record Fig. 7 (the "dual beam 


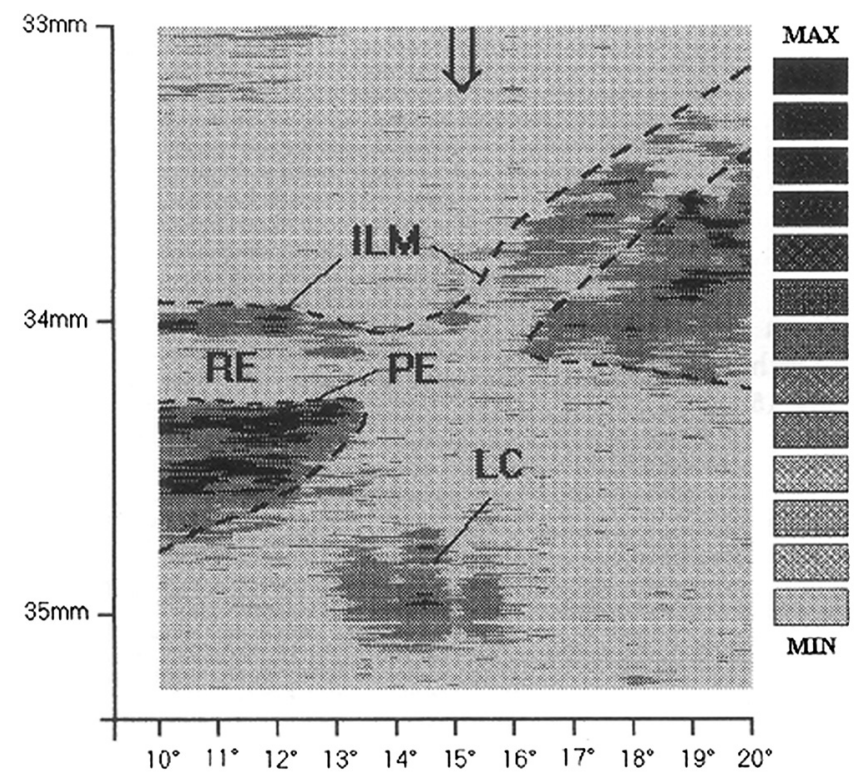

Fig. 7 In vivo retinal image recorded by Fercher's group in 1993. ILM, inner limiting membrane; LC, lamina cribrosa; PE, pigment epithelium; and RE, retina. Reproduced with permission from Ref. 31, ( Elsevier.

technique" that uses the cornea as a reference surface) were inferior to those of the MIT group, Fercher and his group decided to work on a radically new technique intended to overcome previous limitations. This technique, Fourier-domain OCT, eventually led to a paradigm change of OCT technology.

\subsection{Fourier-Domain Optical Coherence Tomography}

Fourier-domain (FD) OCT is based on the Fourier diffraction theorem, which essentially states that the amplitude of the electric field scattered back by a sample is proportional to the (inverse) Fourier transform of the sample scattering potential. ${ }^{33}$ Hence, the sample structure can be reconstructed by a Fourier transform of the spectral distribution of the backscattered field. For FD PCI and OCT, the interfering light fields in the detection arm of the interferometer are therefore recorded as a function of wavenumber, and a Fourier transform of these data provides an $A$-scan. $A$-scans recorded at adjacent positions can then be mounted to 2-D and 3-D images, such as in time-domain (TD) OCT. Since the reference mirror can now be kept at a fixed position, no moving parts are necessary to perform an $A$-scan, and the system can be very fast.

Two alternative technological concepts can be used for FD OCT: (a) the use of a broadband light source in combination with a spectrometer at the interferometer exit [spectral domain (SD) OCT] and (b) the use of a laser with narrow instantaneous linewidth that is rapidly tunable over a large wavenumber range, in combination with a single detector [swept source (SS) OCT]. Both methods are equivalent from a theoretical point of view.

The basic idea of using FD PCI for ocular biometry can already be found in an early paper from 1991 by Fercher et al. ${ }^{34}$ Although the paper deals with TD PCI, the following sentence can be found in the theory section: "Secondly, we can use a spectrometer to evaluate the spectral modulation given by equation (6). Here, $D$ can be obtained from the distance $\delta \omega$ of two dark bands in the channeled spectrum." The first experimental realization was published by Fercher and his group in $1995 .^{5}$ After describing both versions (SD PCI and
SS PCI) in the theoretical section, the spectrometer-based approach is experimentally demonstrated to measure distances within a model eye as well as the thickness of a human cornea in vivo. Figure 8 shows sketches of basic SD [Fig. 8(a)] and SS [Fig. 8(b)] PCI experimental arrangements, as well as the first in vivo $A$-scan acquired by SD PCI [Fig. 8(c)]. The signal peak at $0.77 \mathrm{~mm}$ indicates the posterior surface of the cornea (with reference to the anterior surface), and a division by the group refractive index provides the geometrical thickness. The poor signal quality of the scan was caused by the light source used (multimode laser diode with $\sim 100-\mu$ m coherence length) and by the camera used in these early experiments: a single line of 240 pixels length of an array camera, operating at a frame rate of $\sim 25 \mathrm{~Hz}$, was used. This severely limited the resolution and the phase stability (the latter delayed the discovery of the FD OCT sensitivity advantage). Again, Fercher and his group were ahead of their time. (It should be mentioned in this context that the lack of suitable swept sources also delayed the development of SS-OCT; the first papers demonstrating SS-OCT in phantoms and in vitro were published in 1996 by Haberland et al. ${ }^{35}$ and, with improved resolution, by Chinn et al in $1997,{ }^{36}$ and the first paper demonstrating in vivo SS-PCI for axial eye length measurements in 1997 by Lexer et al. ${ }^{37}$ However, the tunable lasers used in these papers suffered from slow speed and/or a very narrow tuning range; it
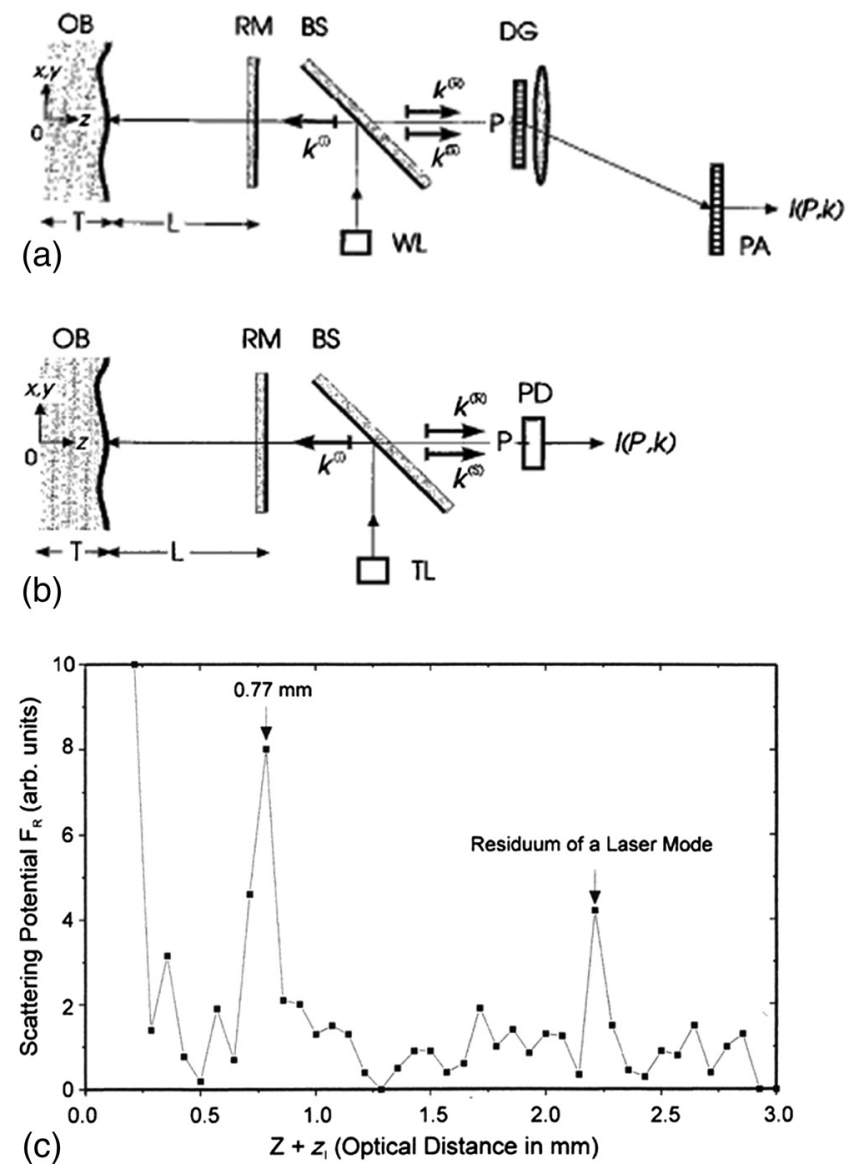

Fig. 8 Fourier-domain $\mathrm{PCl}$. (a) Sketch of spectral-domain $\mathrm{PCl}$, (b) sketch of SS (tunable laser) $\mathrm{PCl}$, (c) measurement of corneal thickness in vivo by an instrument based on (a). BS, beam splitter; $\mathrm{DG}$, diffraction grating; $\mathrm{OB}$, object; $\mathrm{PA}$, photodetector array; PD, photodetector; TL, tunable laser; and $\mathrm{WL}$, white light source. Reproduced with permission from Ref. 5, () Elsevier. 
took more than a decade until swept sources with sufficient speed and bandwidth became commercially available).

The situation dramatically improved with the advent of fast, sensitive, and high-resolution line scan cameras. Leitgeb, who joined Fercher's group in 1998, took over the work on SD PCI and OCT, using high-speed line-scan cameras. In early 2002, in the context of a first demonstration of SD Doppler OCT with a line scan camera operating at $10-\mathrm{kHz}$ line rate, Leitgeb et al. ${ }^{38}$ first presented equations for the signal-to-noise ratio of SD OCT in the shot noise limit. Compared with TD OCT, they predicted a huge sensitivity advantage of $\sim 18 \mathrm{~dB}$ for the configuration used. Later in that year, Wojtkowski (Nicolaus Copernicus Univ., Torun, Poland), who had worked during his study for several months in Fercher's group, demonstrated the first in vivo retinal SD OCT images in a joint publication with the Vienna group. ${ }^{39}$ These images clearly indicated a sensitivity superior to what would have been possible by TD OCT for the same settings of illumination power and data acquisition speed.

In April 2003, Leitgeb et al. ${ }^{40}$ published a comprehensive theoretic derivation of the FD OCT sensitivity advantage, including shot noise, excess noise, and receiver noise terms, together with an experimental demonstration of the theoretic results. Figure 9 shows the theoretic sensitivity curves (sensitivity versus reference arm reflectivity), calculated for FD and TD OCT for the experimental conditions used in that work, as well as the experimentally measured sensitivity. Theoretic predictions and experimental results are in good agreement. The parallel detection scheme of FD OCT theoretically improves the sensitivity by a factor proportional to the number of pixels in the line scan camera; in practice, improvements by 2 to 3 orders of magnitude are nowadays achievable. Two other research groups independently arrived at the same results shortly thereafter. ${ }^{41,42}$ Together, these papers led to a paradigm change of OCT technology, with FD OCT methods largely replacing the first-generation TD technique. FD OCT, based on Fercher's original idea, is one of the most successful biomedical optics techniques developed so far, with more than a dozen companies on the market and 10,000 s of instruments installed worldwide.

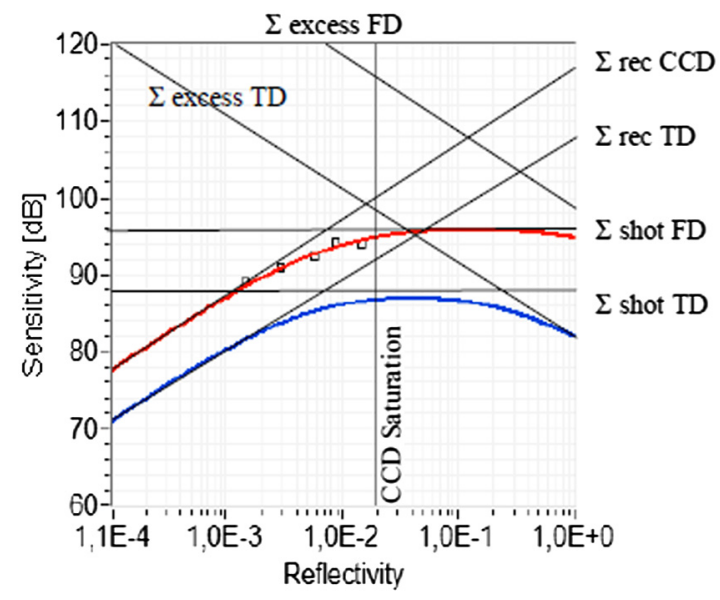

Fig. 9 Sensitivity advantage of Fourier-domain OCT versus timedomain OCT. Plot of sensitivity as a function of reference arm reflectivity. Red curve: theoretic plot of FD OCT sensitivity; blue curve: theoretic plot of TD OCT sensitivity; $\Sigma$ excess: theoretic excess noise limit; $\Sigma$ rec: theoretic receiver noise limit; $\Sigma$ shot: theoretic shot noise limit; and squares: measured sensitivity with an FD OCT setup. Reproduced with permission from Ref. 40, (c) Optical Society of America.

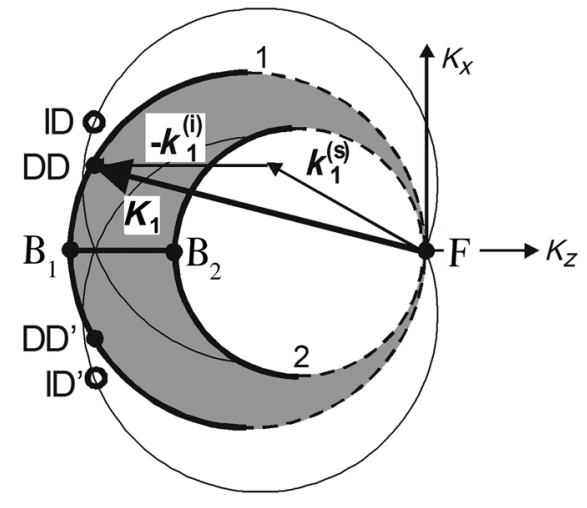

Fig. 10 Ewald sphere representation of backscattering geometry in OCT signal generation. $F, B_{1,2}$, Fourier data obtained by forward and backward scattering; DD and $\mathrm{DD}^{\prime}$, Fourier components obtained by detector position diversity; ID and ID', Fourier components obtained by illumination direction diversity. Reproduced with permission from Ref. 33, () IOP Publishing.

\subsection{Contributions to Optical Coherence Tomography Theory}

In addition to the seminal work on FD OCT and its principles, Fercher and his group contributed to many other aspects of OCT theory and technology, such as single- and multiple scattering, speckle, dispersion, resolution, sensitivity, and full range complex OCT. A discussion of all this work would be beyond the scope of this paper. More details can be found in various review papers and book chapters (see, e.g., Refs. 33 and 43-46).

As an example of the approach to OCT principles that is characteristic of Fercher's fundamental way of analysis of which information is accessible by OCT, and of how this information might be expanded, his analysis of the backscattering geometry of OCT signal acquisition should be mentioned. ${ }^{33}$ Using the Ewald sphere representation (cf. Fig. 10), Fercher was able to show that all Fourier components of the scattering potential accessible by scattering in different directions are located in the gray-shaded area between two spheres spanned by the arrowheads of the scattering vectors $\mathbf{K}$ corresponding to the wavelength band emitted by the OCT light source. Standard OCT normally operates in backscattering mode, so only the Fourier components along the line B1 to B2 are accessible. This immediately leads to the high-pass nature of OCT signals: only areas of rapidly changing refractive index give rise to an OCT signal; areas of slowly varying refractive index remain invisible. Moreover, only a tiny fraction of the Fourier components (and information on the corresponding components of the object scattering potential) is accessible by direct backscattering. Fercher now argued that, by placing detectors at other positions, such as DD or $\mathrm{DD}^{\prime}$ (or by illuminating from other directions, e.g., ID and $\mathrm{ID}^{\prime}$ ), other Fourier components, corresponding to other backscattering angles, become accessible. This provides additional information on the sample. In recent years, new approaches of angle-resolved OCT and multibeam OCT exploit these concepts. ${ }^{47-51}$

\section{Teaching}

In addition to his seminal research work, Fercher was also an enthusiastic and dedicated teacher. Appointed as professor of medical physics, he taught courses on basic physics for undergraduate students of medicine as well as courses on medical 
physics for postgraduate students. He had a comprehensive knowledge of all aspects of physics and their relation to medical issues, stretching from biomechanics over thermodynamics, electromagnetics, and optics to radiation physics. In his lectures, he strived to demonstrate the medical implications of basic/ theoretic physical concepts, be they related to basic biologic functions of the human body or to medical technology. In this context, it should also be mentioned that Fercher was very interested in other (nonoptical) noninvasive medical imaging techniques, especially in MR imaging, which led to his enthusiastic support of the MR imaging group in the Department of Medical Physics. More details on Fercher's teaching activities would be beyond the scope of this review; however, more insights into his concepts of medical physics teaching can be found in his comprehensive book "Medizinische Physik.,"52

\section{Recognition}

The ISI Web of Science lists a total of $\sim 110$ papers authored or coauthored by Fercher in peer-reviewed journals. These papers have a total citation count of $\sim 10,500$ citations or, on average, 95 citations per paper. This extraordinarily high citation count per paper is very unusual and underlines his broad recognition in the scientific community.

Unfortunately, Fercher had to wait a long time, until after his retirement, to receive the recognition he deserved in terms of awards or prizes. In 2014, he received the Combined Royal Colleges Medal of the Royal Photographic Society and, in 2017, the Fritz J. and Dolores H. Russ Prize of the US National Academy of Engineering (jointly with J.G. Fujimoto, D. Huang, E.A. Swanson, and the author of this review) "for optical coherence tomography, leveraging creative engineering to invent imaging technology essential for preventing blindness and treating vascular and other diseases." A few weeks after the awards ceremony, Fercher passed away.

\section{Legacy}

Fercher was one of the true pioneers of biomedical optics. His legacy to the scientific community is manifold: with the first demonstration of PCI in ocular tissue, he laid the cornerstone of OCT. PCI intraocular ranging, based on his original idea, has revolutionized ocular biometry for cataract surgery, and, with $10,000 \mathrm{~s}$ of optical biometry systems installed worldwide, it is now the gold standard for this application. Spectral-domain OCT, first devised by Fercher and his group, led to a paradigm change in OCT technology. Again, with 10,000 s of FD OCT systems installed throughout the globe, it is now the gold standard for retinal diagnostics.

Furthermore, the formation of the Vienna Biomedical Optics Group should be mentioned here. This group, which later subdivided into several groups led by independent, internationally renowned researchers who still have close connections and cooperations, is certainly an important legacy. In this context, the OCT group at Nicolaus Copernicus University in Torun, whose formation received strong support by Fercher and his group, especially in the initial phase, also should be mentioned.

Finally, his many scientific papers may contain many more ideas that might be worth being explored. As mentioned, Fercher often was way ahead of his time, and it is certainly worth studying his papers again, to think about the concepts and to explore them with modern technology. This may lead to future breakthroughs in the field of OCT and would be in the best of his spirit.

\section{Disclosures}

The author declares that there are no conflicts of interest.

\section{Acknowledgments}

The author of this paper is grateful to A.F. Fercher for introducing him to the field of biomedical optics, his mentorship in the early phase, and countless discussions on various aspects of optics and medical physics over the past 30 years. The author also acknowledges valuable discussions with W. Drexler, R. Leitgeb, E. Moser, M. Pircher, L. Schmetterer, and I. Strasser from the Vienna group, as well as with R. Birngruber, T. Lasser, and M. Wojtkowski.

\section{References}

1. A. F. Fercher and J. D. Briers, "Flow visualization by means of singleexposure speckle photography," Opt. Commun. 37(5), 326-330 (1981).

2. A. F. Fercher et al., "Eye deformation measurement by laser interferometry," Opt. Acta: Int. J. Opt. 29(10), 1401-1406 (1982).

3. A. F. Fercher and E. Roth, "Ophthalmic laser interferometry," Proc. SPIE 658, 48 (1986).

4. A. F. Fercher, K. Mengedoht, and W. Werner, "Eye-length measurement by interferometry with partially coherent-light," Opt. Lett. 13(3), 186188 (1988).

5. A. F. Fercher et al., "Measurement of intraocular distances by backscattering spectral interferometry," Opt. Commun. 117(1-2), 43-48 (1995).

6. C. K. Hitzenberger et al., "Key developments for partial coherence biometry and optical coherence tomography in the human eye made in Vienna," Invest. Ophthalmol. Visual Sci. 57(9), OCT460-OCT474 (2016).

7. A. F. Fercher, "Computation of diffraction patterns for biological cell models based on Mie theory," Optik 43(2), 129-148 (1975).

8. J. D. Briers and A. F. Fercher, "Retinal blood-flow visualization by means of laser speckle photography," Invest. Ophthalmol. Visual Sci. 22(2), 255-259 (1982).

9. A. F. Fercher, M. Peukert, and E. Roth, "Visualization and measurement of retinal blood-flow by means of laser speckle photography," Opt. Eng. 25(6), 731-735 (1986).

10. A. K. Dunn et al., "Dynamic imaging of cerebral blood flow using laser speckle," J. Cereb. Blood Flow Metab. 21(3), 195-201 (2001).

11. D. A. Boas and A. K. Dunn, "Laser speckle contrast imaging in biomedical optics," J. Biomed. Opt. 15(1), 011109 (2010).

12. T. Sugiyama et al., "Use of laser speckle flowgraphy in ocular blood flow research," Acta Ophthalmol. 88(7), 723-729 (2010).

13. http://www.softcare-ltd.co.jp/index.en.html.

14. A. F. Fercher and J. D. Briers, "Eye deformation measurement by laser interferometry," Proc. SPIE 369, 42 (1983).

15. A. F. Fercher, "In vivo measurement of fundus pulsations by laser interferometry," IEEE J. Quantum Electron. 20(12), 1469-1471 (1984).

16. L. F. Schmetterer et al., "Topical measurement of fundus pulsations," Opt. Eng. 34(3), 711-716 (1995).

17. L. Schmetterer and M. Wolzt, "Laser interferometric investigations of pulsatile choroidal blood flow: review and new results on the validity of the technique," J. Biomed. Opt. 3(3), 246-252 (1998).

18. L. Schmetterer et al., "Effects of antiglaucoma drugs on ocular hemodynamics in healthy volunteers," Clin. Pharmacol. Ther. 61(5), 583595 (1997).

19. L. Schmetterer and M. Wolzt, "Ocular blood flow and associated functional deviations in diabetic retinopathy," Diabetologia 42(4), 387-405 (1999).

20. A. F. Fercher, Verfahren und Anordnung zur Messung der Teilstrecken des lebenden Auges, Deutsches Patentamt, Germany (1983).

21. C. K. Hitzenberger, "Optical measurement of the axial eye length by laser Doppler interferometry," Invest. Ophthalmol. Visual Sci. 32(3), 616-624 (1991).

22. C. K. Hitzenberger, "Measurement of corneal thickness by low-coherence interferometry," Appl. Opt. 31(31), 6637-6642 (1992). 
23. W. Drexler et al., "Measurement of the thickness of fundus layers by partial coherence tomography," Opt. Eng. 34(3), 701-710 (1995).

24. W. Drexler et al., "Biometric investigation of changes in the anterior eye segment during accommodation," Vision Res. 37(19), 2789-2800 (1997).

25. W. Drexler et al., "Eye elongation during accommodation in humans: differences between emmetropes and myopes," Invest. Ophthalmol. Visual Sci. 39(11), 2140-2147 (1998).

26. C. K. Hitzenberger et al., "Measurement of the axial length of cataract eyes by laser-Doppler interferometry," Invest. Ophthalmol. Visual Sci. 34(6), 1886-1893 (1993).

27. W. Drexler et al., "Partial coherence interferometry: a novel approach to biometry in cataract surgery," Am. J. Ophthalmol. 126(4), 524-534 (1998).

28. O. Findl et al., "Improved prediction of intraocular lens power using partial coherence interferometry," J. Cataract Refractive Surg. 27(6), 861-867 (2001).

29. https://www.zeiss.com/meditec/int/products/ophthalmology-optometry/ cataract/diagnostics/optical-biometry/iolmaster-500.html.

30. D. Huang et al., "Optical coherence tomography," Science 254(5035), 1178-1181 (1991).

31. A. F. Fercher et al., "In-vivo optical coherence tomography," Am. $J$. Ophthalmol. 116(1), 113-114 (1993).

32. J. A. Izatt et al., "Ophthalmic diagnostics using optical coherence tomography," Proc. SPIE 1877, 136 (1993).

33. A. F. Fercher et al., "Optical coherence tomography-principles and applications," Rep. Prog. Phys. 66(2), 239-303 (2003).

34. A. F. Fercher, C. Hitzenberger, and M. Juchem, "Measurement of intraocular optical distances using partially coherent laser-light," J. Mod. Opt. 38(7), 1327-1333 (1991).

35. U. Haberland, V. Blazek, and H. J. Schmitt, "Optical coherent tomography with electrically tunable semiconductor laser using FMCW techniques," Proc. SPIE 2925, 227 (1996).

36. S. R. Chinn, E. A. Swanson, and J. G. Fujimoto, "Optical coherence tomography using a frequency-tunable optical source," Opt. Lett. 22(5), 340-342 (1997).

37. F. Lexer et al., "Wavelength-tuning interferometry of intraocular distances," Appl. Opt. 36(25), 6548-6553 (1997).

38. R. Leitgeb et al., "Flow velocity measurements by frequency domain short coherence interferometry," Proc. SPIE 4619, 16 (2002).

39. M. Wojtkowski et al., "In vivo human retinal imaging by Fourier domain optical coherence tomography," J. Biomed. Opt. 7(3), 457463 (2002).
40. R. Leitgeb, C. K. Hitzenberger, and A. F. Fercher, "Performance of Fourier domain vs. time domain optical coherence tomography," Opt. Express 11(8), 889-894 (2003).

41. J. F. de Boer et al., "Improved signal-to-noise ratio in spectral-domain compared with time-domain optical coherence tomography," Opt. Lett. 28(21), 2067-2069 (2003).

42. M. A. Choma et al., "Sensitivity advantage of swept source and Fourier domain optical coherence tomography," Opt. Express 11(18), 21832189 (2003).

43. A. F. Fercher, "Optical coherence tomography," J. Biomed. Opt. 1, 157-173 (1996).

44. A. F. Fercher and C. K. Hitzenberger, "Optical coherence tomography," Prog. Opt. 44, 215-302 (2002).

45. A. F. Fercher, "Optical coherence tomography-development, principles, applications," Z. Med. Phys. 20(4), 251-276 (2010).

46. A. F. Fercher, "Inverse scattering and aperture synthesis in OCT," in Optical Coherence Tomography-Technology and Applications, W. Drexler and J. G. Fujimoto, Eds., pp. 143-164, Springer, Cham (2015).

47. A. Wax et al., "Measurement of angular distributions by use of lowcoherence interferometry for light-scattering spectroscopy," Opt. Lett. 26(6), 322-324 (2001).

48. Y. Yang et al., "Quantitative analysis of angle-resolved scattering properties of ovarian tissue using optical coherence tomography," J. Biomed. Opt. 17(9), 090503 (2012).

49. R. M. Werkmeister et al., "Bidirectional Doppler Fourier-domain optical coherence tomography for measurement of absolute flow velocities in human retinal vessels," Opt. Lett. 33(24), 2967-2969 (2008).

50. W. Trasischker et al., "In vitro and in vivo three-dimensional velocity vector measurement by three-beam spectral-domain Doppler optical coherence tomography," J. Biomed. Opt. 18(11), 116010 (2013).

51. A. Wartak et al., "Active-passive path-length encoded (APPLE) Doppler OCT," Biomed. Opt. Express 7(12), 5233-5251 (2016).

52. A. F. Fercher, Medizinische Physik: Physik für Mediziner, Pharmazeuten und Biologen, Springer, Wien, New York (1999).

Christoph K. Hitzenberger is a professor of medical physics and vice chair of the Center for Medical Physics and Biomedical Engineering, Medical University of Vienna, and an editor-in-chief of Biomedical Optics Express. He works in biomedical optics since 1987 and is one of the pioneers of low coherence ocular biometry and optical coherence tomography. He is a fellow of SPIE and of OSA. His pioneering contributions to OCT were awarded with the Russ Prize of the National Academy of Engineering in 2017. 